\title{
Analyser du discours : le cas des débats politiques télévisés
}

\author{
Kerbrat-Orecchioni, Catherine \\ ICAR \& Université Lumière Lyon 2 \\ catherine.kerbrat-orecchioni@univ-lyon2.fr
}

\section{Préliminaires}

On appelle «analyse du discours » la branche des sciences du langage dont l'objet est constitué d'échantillons attestés de productions langagières. Mais en quoi consiste l'activité d' "analyse », s'agissant d'un tel objet ou plutôt, d'objets aussi divers ?

Les différentes formes de discours présentent des points communs liés à leur essence langagière, mais aussi toutes sortes de spécificités qui impliquent le recours à des méthodologies diversifiées. L'énorme masse des discours attestés dans une société donnée peut être typologisée sur la base de nombreux critères, principalement et entre autres : le caractère écrit ou oral du discours soumis à l'analyse, et son caractère dialogué ou non dialogué. Nous dirons pour commencer quelques mots de ces deux dimensions, envisagées par rapport à ce qui constitue ici notre objet propre : les débats politiques télévisées se déroulant en contexte électoral, et plus précisément les débats de l'entre-deux-tours des élections présidentielles françaises de la cinquième République (soit cinq à ce jour : 1974, 1981, 1988, 1995, 2007 ${ }^{1}$ ), ces «rituels républicains » (Delporte 2012) inaugurés en 1974 avec le débat GiscardMitterrand et dont les règles de fonctionnement se sont stabilisées à partir du débat de 1981 .

\subsection{Deux distinctions fondamentales pour une typologie des discours}

\subsubsection{Discours oral vs écrit}

La langue a cette particularité qu'elle peut se réaliser sous deux formes, orale et écrite, entre lesquelles il n'existe aucun continuum: ou le matériau utilisé est de nature phonique, ou il est de nature graphique. On ne peut pas, en ce sens, parler de discours « intermédiaires » entre l'oral et l'écrit, même s'il est vrai que certains types de discours écrits (comme les chats) possèdent certaines propriétés de l'oral, et inversement (il arrive qu'on parle «comme un livre»); que la communication peut mobiliser simultanément du matériel écrit et oral : les interactions mixtes (« oralographiques ») sont fréquentes, en particulier en contexte didactique (Bouchard 2005) mais aussi dans certaines situations de la vie quotidienne ; qu'enfin les deux systèmes sont mutuellement convertibles : un texte écrit peut être oralisé tout comme un discours oral peut être scripturalisé, ces opérations de «transsubstantiation » s'accompagnant nécessairement de modifications plus ou moins importantes du message d'origine (ce n’est pas seulement sa matérialité qui se trouve affectée).

En ce qui concerne nos données : il s'agit de discours oraux, à caractère « multimodal » ${ }^{2}$, c'est-à-dire plurisémiotique (exploitation simultanée d'unités linguistiques, voco-prosodiques et mimo-gestuelles) et multicanal (ils empruntent à la fois les canaux auditif et visuel). Mais pour pouvoir être analysé le discours oral doit être «transcrit », c'est-à-dire transformé en un objet graphique, l'analyse s'effectuant dans un va-et-vient constant entre cet artefact et l'enregistrement lui-même, c'est-à-dire entre ce que l'on appelle parfois ${ }^{3}$ les données «primaires » (l'enregistrement de l'événement) et « secondaires » (sa transcription), étant donné que l'on n'a pas accès aux données « brutes » (l'événement lui-même) et que nous sommes tributaires de la prise de vue effectuée par l'équipe de tournage conformément à la réglementation en vigueur (elle impose par exemple, comme il est rappelé au début du débat de 1988, de ne faire apparaître à l'écran que le locuteur en place, ce qui nous prive des réactions mimiques éventuelles de son interlocuteur). 


\subsubsection{Discours « monologal " vs « discours en interaction »}

Tous les discours ont une dimension «dialogique », dans la mesure où ils prennent en compte un destinataire supposé et où ils incorporent en outre généralement d'autres voix énonciatives que celle du locuteur/scripteur. Mais pour que l'on puisse parler véritablement d'interaction, encore faut-il que se trouvent en présence deux participants (ou plus) en chair et en os, qui co-construisent en temps réel une sorte de «texte » en ajustant au coup par coup leurs comportements mutuels, et en négociant tout au long du déroulement de l'interaction les divers aspects de son fonctionnement ${ }^{4}$.

Cette distinction recouvre en grande partie la précédente, mais en partie seulement car il existe de nos jours des formes d'écrit véritablement interactives et à l'inverse, certaines formes d'oral ne sont que faiblement interactives - ce deuxième axe est en effet graduel : il faut admettre l'existence de degrés d'interactivité, le degré maximal étant représenté par les conversations familières. En ce qui concerne nos données : s'il est vrai qu'il ne s'agit pas vraiment de fresh talk (Goffman 1981 : 171-172) étant donné que les débatteurs ont largement préparé leurs interventions sous la houlette de leurs conseillers en communication, la dynamique du débat laisse une large part à l'improvisation; et les échanges font alterner de longues tirades « monologales » avec des passages où l'alternance des tours de parole se fait à un rythme accéléré, avec multiplication des interruptions et chevauchements, ces «zones de turbulence » étant de plus en plus fréquentes avec l'évolution du genre (des cinq débats constituant notre corpus c'est assurément celui de 2007 qui présente le plus fort degré d'interactivité).

\subsection{Implications méthodologiques}

Etant donné les propriétés constitutives des discours qui nous intéressent, leur analyse doit impérativement obéir aux principes suivants :

(1) Nécessité de prendre en compte la totalité du matériel qui les compose (mimogestualité comprise) et de travailler à partir d'une transcription fidèle des données ${ }^{5}$.

(2) Nécessité de prendre en compte le caractère co-construit du discours, c'est-à-dire d'être attentif, pour tout segment analysé, à ce à quoi il réagit en amont et à la réaction qu'il déclenche en aval, ainsi qu'à l'ensemble des influences mutuelles qu'exercent les participants au débat tout au long de son déroulement.

(3) À ces deux exigences j'ajouterai celle de prendre en compte les facteurs contextuels les plus évidemment pertinents, en relation avec le genre interactionnel dont relève le discours analysé : nature du dispositif et du format participatif (complexe dans le cas des débats médiatiques), enjeux discursifs et objectifs des participants, aux niveaux à la fois local (où il s'agit de produire pas à pas des enchaînements appropriés) et global - dans le cas des débats électoraux qui nous intéressent, les activités des débatteurs sont entièrement tributaires de cette visée surplombante : l'emporter sur l'adversaire dans le débat, en espérant l'emporter ensuite dans les urnes... Décrire ce qui se passe « du point de vue des membres ", cela consiste entre autres, à l'instar de l'ensemble des participants à l'événement communicatif, à ne jamais perdre de vue cet objectif.

Il n'en reste pas moins que ces débats peuvent être abordés de bien des façons, qui sont complémentaires et également légitimes : on peut travailler sur des unités plus ou moins étendues, opter pour une approche quantitative ou qualitative, adopter une perspective comparative ou non, se focaliser sur divers aspects des débats en mobilisant des outils descriptifs également divers... ${ }^{6}$ Pour ce qui nous concerne, nous nous attacherons à la description de quelques séquences dont tous les commentateurs admettent qu'elles correspondent à des épisodes particulièrement « marquants » au sein de cet ensemble constitué des cinq «duels » de l'entre-deux-tours des présidentielles. Ces séquences sont de dimension variable (à l'exception de la dernière, il s'agit de ce que l'on a aujourd'hui coutume d'appeler des 《 petites phrases $\rangle^{7}$ ), mais elles ont en commun les propriétés d'être « détachables » et «mémorables », au point de s'être inscrites dans la mémoire collective sous une forme relativement autonome par rapport au contexte qui leur a donné naissance. 
Nous en avons retenu une par débat, à savoir ${ }^{8}$ :

(1) Débat Giscard-Mitterrand de 1974, Valéry Giscard d'Estaing: «Vous n'avez pas monsieur Mitterrand le monopole du cœur »;

(2) Débat Giscard-Mitterrand de 1981, François Mitterrand : «Je ne suis pas votre élève et vous n'êtes pas le président de la République ici »;

(3) Débat Chirac-Mitterrand de 1988, François Mitterrand : « Mais vous avez tout à fait raison monsieur le Premier ministre »;

(4) Débat Chirac-Jospin de 1995, Lionel Jospin : «Il vaut mieux cinq ans avec Jospin que sept ans avec Jaques Chirac »;

(5) Débat Royal-Sarkozy de 2007, Ségolène Royal : « Je ne suis pas énervée je suis en colère, ce n'est pas pareil ».

Au terme de cette description on se demandera sur quoi repose le caractère «marquant» de ces séquences mais au-delà, on reviendra sur notre questionnement de départ : que fait-on exactement quand on « analyse du discours », et plus spécifiquement du discours en interaction ? Quels types de savoirs et quelles ressources interprétatives doit-on mobiliser pour mener à bien cette entreprise ? Si l'on admet que l'analyste doit s'efforcer, ainsi que le préconise l'analyse conversationnelle, d'adopter « le point de vue des membres », comment la formule s'applique-t-elle au cas particulier des discours médiatiques et en particulier, quelle place convient-il d'accorder dans la description à ces millions de récepteurs qui bien que n'intervenant pas directement dans le débat n'en constituent pas moins les principaux destinataires, et les dépositaires de cette mémoire collective qui retient certains épisodes comme particulièrement « marquants»?

\section{Analyses}

\subsection{4 : débat entre Valéry Giscard d'Estaing et François Mitterrand}

- VGE : «Vous n'avez pas monsieur Mitterrand le monopole du cœur»

Dans ce premier face-à-face télévisé entre les deux finalistes d'une élection présidentielle, Valéry Giscard d'Estaing (VGE), ministre de l'Economie et des Finances depuis 1969, affronte François Mitterrand (FM), candidat de l'Union de la gauche. Celui-ci répète sur tous les tons que le gouvernement de droite auquel appartient son adversaire mène une politique «antisociale » favorisant exclusivement une «caste» de privilégiés; accusation que VGE réfute dans de longues tirades argumentées, mais aussi dans un premier échange un peu vif où il adopte une posture indignée, interrompant FM qu'il contraint à faire machine arrière :

FM : [...] voyez vous/ le le changement sans risque/ dont vous avez parlé $\backslash$ (.) il est sans risque/ naturellement/pour des gens comme vous/ (.) mais pensez maintenant/ (.)

VGE : qu'appelez vous/monsieur Mitterrand/ des gens comme vous

FM : c'est-à-dire des gens qui appartiennent/ à une certaine caste sociale/ (.) qui n'ont pas/ en effet/ à se trouver/ (.) affrontés/ (.) comme le sont la plupart des des des femmes et des hommes/

[qui ont voté pour moi/]

VGE : [monsieur Mitterrand/ vous n'avez pas le droit/ de dire des choses pareilles $\backslash$ [...] je vous interromps [...] [nous représentons nos électeurs/

FM : $\quad$ [exactement exactement/

VGE : je représente des électeurs/ qui sont aussi modestes/que les vôtres/ [...] 
Mais le passage qui nous intéresse survient plus tard (au bout d'une heure et quart de débat). Il est déclenché par une nouvelle salve de FM contre l'injustice de la politique fiscale menée par son adversaire, au cours de laquelle il introduit le thème du « cœur », accusant son adversaire d'en manquer et faisant de cette injustice un scandale non seulement intellectuel mais moral. VGE va alors s'emparer de ce mot de « cœeur » pour infliger à son adversaire un sévère coup de semonce :

FM : $\quad[. .$.$] je dirai/ c'est presque une question/ (.) naturellement d'intelligence/ (.) c'est aussi une affaire$ de cour/ (.) il n'est pas acceptable/ qu'il y ait une petite catégorie/ de privilégiés/ (.) qui sont/ qui s-/ qui sont servis par toutes vos lois/ en particulier/ par (.) vos lois fiscales/ (.) qui se tirent de tout/ qui (.) reçoivent/ des (.) des jetons de présence/ des tantièmes/ des bénéfices dans les conseils d'administration/ (.) des sommes énormes/ et scandaleuses/ (.) que la plupart des/ (.) des grandes sociétés/ (.) aient des bénéfices/ (.) SANS comparaison/ possible/ (.) avec qui que ce soit d'autre/ tandis que TANT de millions de gens/ vivent difficilement/ [...] et c'est LÀ/ (.) que j'engage/ (.) ma candidature $\backslash$ (.) $[. .$.$] c'est ma candidature/ et mon programme/ (.) d'action/ (.) qui permettront$ demain/ (.) à la France/ d'engager la grande aventure/ l'admirable/ aventure $\backslash$ (.) non seulement de la réussite économique/ (.) mais aussi du progrès social $\backslash$ c'est là/ (.) tout notre débat/ (.)

JB (Jacqueline Baudrier) : mon [sieur Giscard d'Estaing/

VGE :

[hé bien monsieur Mitterrand/ si on vous a écouté/ comme je vous ai écouté $\backslash$ avec intérêt $\backslash$ (.) euh on est convaincu que ce qu'il faut faire/ ça n'est pas ce que vous proposez (.) d'abord/ je vais vous dire quelque chose/ je trouve toujours/ (.) choquant/ (.) et blessant $\backslash$ (.) de s'arroger/ (.) le monopole du cœur $\backslash$ (.) vous n'avez pas/ monsieur Mitterrand le monopole du cœur\vous ne l'avez pas

FM : sûrement pas $\backslash$

VGE : euh j'ai un cour/ comme le vôtre/ qui bat à sa cadence/ et qui est le mien $\backslash$ (.) vous n'avez pas/ le monopole du cœur $($.) et ne parlez pas aux Français/ euh de cette façon/ (.) euh si/ (.) blessante pour les autres $\backslash$ (.) alors concernant/ ce qui a été fait au cours des dernières années $\backslash$ (.) [...]

Encadrée par les unités démarcatives «d'abord» et «alors», préfacée par «je vais vous dire quelque chose » qui accentue son caractère solennel, cette déclaration interrompt provisoirement le cours du débat par une mise au point censée lui permettre de se poursuivre sur des bases plus saines.

Cette attaque frontale (emploi de la deuxième personne et du terme d'adresse, ton de remontrance, regard maintenu sur l'adversaire, mimique sévère) se caractérise par son élaboration rhétorique et son organisation quasiment musicale (thème avec reprises et variations). Elle débute avec l'énoncé d'un principe général mais formulé sur un mode éminemment «subjectif»: mobilisation du modalisateur «je trouve » ainsi que de deux adjectifs fortement axiologisés et mis en valeur prosodiquement, le premier («choquant») renvoyant plutôt à un scandale intellectuel et le second («blessant») à un état affectif. Puis c'est l'introduction du syntagme «monopole du cœur » qui sera répété quatre fois (dont l'une sous la forme d'un pronom anaphorique), ce qui vient encore renforcer le poids que confère à l'énoncé central ( « vous n'avez pas monsieur Mitterrand le monopole du cœur ») sa structure syntaxique et rythmique (succession de trois segments de dimension croissante, composés respectivement de quatre, cinq et six syllabes).

À ce petit sermon FM peut difficilement faire autre chose qu'acquiescer («sûrement pas » prononcé hors champ). Mais VGE enfonce le clou par une reformulation qui relève à la fois d'une évocation très fortement chargée émotionnellement et d'une sorte d'étayage argumentatif: vous n'avez pas le monopole du cœur, puisque j'en ai un moi aussi - argument imparable, mais qui repose en réalité sur l'exploitation de la polysémie du mot «cœur », lequel désigne à la fois l'organe et par métonymie, les sentiments dont cet organe est censé être le siège, comme la générosité et l'aptitude à la compassion ; mais comme il n'est pas évident que ces sentiments soient aussi universellement partagés que l'élément anatomique, on a affaire à une argumentation en trompe l'œil (j'ai $d u$ cœur puisque j'ai $u n$ cœur). En dépit de ses allures de sophisme, l'évocation concrète de ce cœur que l'on entend battre «à sa cadence », dans une phrase évoquant à la fois la communauté des deux partenaires et la singularité de chacun $^{9}$, produit un puissant effet émotionnel. Vient enfin la conclusion du raisonnement. Elle prend la 
forme d'une sommation (accompagnée par un hochement de tête de bas en haut qui renforce l'effet « donneur de leçons ») dans laquelle VGE reprend l'adjectif « blessant » mais avec un élargissement de son champ d'application : il associe à sa blessure personnelle les Français « autres », c'est-à-dire tous ceux que le discours de Mitterrand ostracise en les excluant de la communauté des humains.

La parenthèse se referme, et face à un adversaire silencieux VGE enchaîne sur un tout autre ton en justifiant sa politique passée. Mais à la fin de cet exposé il se permet en guise conclusion une petite piqûre de rappel :

donc/ (.) monsieur Mitterrand $\backslash$ (.) personne n'a le monopole du cœur monsieur Mitterrand $\backslash$ (.) personne n'a le monopole de la justicel

On dira pour conclure que l'efficacité de cette intervention de Giscard d'Estaing repose à la fois sur son contenu (elle part en guerre contre une certaine "doxa», que Mitterrand reprend lourdement à son compte et qui consiste à opposer la gauche généreuse à la droite enfermée dans son égoïsme de classe), sur sa formulation, et sur l'ethos qu'elle construit du locuteur (dont on a vu qu'il mobilisait aussi le $\log o s$ et le pathos); un ethos qui va à l'encontre de l'image qui s'attache habituellement à ce personnage, perçu non seulement comme un homme de droite mais aussi comme un économiste compétent mais froid, distant et coupé du peuple (son patronyme n'étant pas fait pour arranger les choses). Giscard s'emploie donc ici à lutter contre ce calamiteux « ethos préalable », ainsi qu'il l'avait déjà fait peu auparavant lors d'une déclaration télévisée ${ }^{10}$ qui se concluait ainsi :

On me fait parfois le reproche d'être quelqu'un de froid. Je crois que ce n'est pas vrai. Je crois que je suis quelqu'un de réservé comme sans doute, d'ailleurs, beaucoup d'entre vous. Mais vous savez que les gens réservés ne sentent pas moins que les autres. C'est pourquoi, dans cette campagne, j'ai dit que je voulais regarder la France au fond des yeux, mais je voudrais aussi atteindre son cœur.

En même temps qu'elle construit de son auteur un ethos compassionnel (et corrélativement, de son adversaire un ethos sectaire), cette sortie giscardienne produit sur l'auditoire un effet " pathétique » par l'irruption inattendue d'un corps et d'un cœur, dont elle fait entendre le battement au sein d'un débat jusqu'alors plutôt désincarné.

\subsection{1 : débat entre Valéry Giscard d'Estaing et François Mitterrand}

- $F M$ : «Je ne suis pas votre élève et vous n'êtes pas le président de la République ici »

Les débatteurs sont ici les mêmes mais le statut de Giscard d'Estaing a entre-temps changé : il est devenu président de la République. Cette donnée est en principe non pertinente dans ce contexte, où les deux candidats sont censés s'affronter à égalité. Mitterrand veille de façon sourcilleuse au respect de ce principe, soucieux de ne pas se laisser traiter de façon condescendante par son adversaire (le risque est particulièrement grand lorsque le débat porte sur des questions économiques et financières, qui sont par excellence le domaine de compétence de VGE). Il prend la mouche une première fois pour reprocher ironiquement à son partenaire son style doctoral :

FM : [...] ces deux chocs pétroliers/ pffff (.) c'est vrai/ (.) bien entendu/ (.) c'est pas la peine de prendre

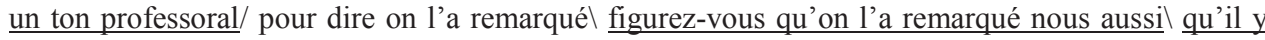
avait eu deux chocs pétroliers $\backslash$ (.)

Mais la coupe déborde lorsque VGE le soumet à une sorte de «colle » en lui posant une question à valeur de vérification de connaissance, accompagnée de cette mimique d'attente caractéristique des examinateurs interrogeant un cancre. FM explose alors : il remet les choses en place et Giscard à sa place (celle d'un simple «contradicteur») en s'offrant le luxe de ne pas fournir immédiatement la réponse à la question posée, tout en administrant in fine la preuve qu'il la connaît bel et bien :

VGE : [...] mais ça ne se passe pas comme ça/ monsieur Mitterrand $\backslash$ actuellement/ (.) du seul fait de nos incertitudes politiques $\backslash$ (.) nous sommes au plancher $\backslash$ (.) et donc il faut agir/ nous agissons/ à l'heure actuelle $\backslash$ (.) nous sommes passés/ (.) comme vous le savez/ pour le Deutsche Mark/ pouvez vous me dire les chiffres/ 


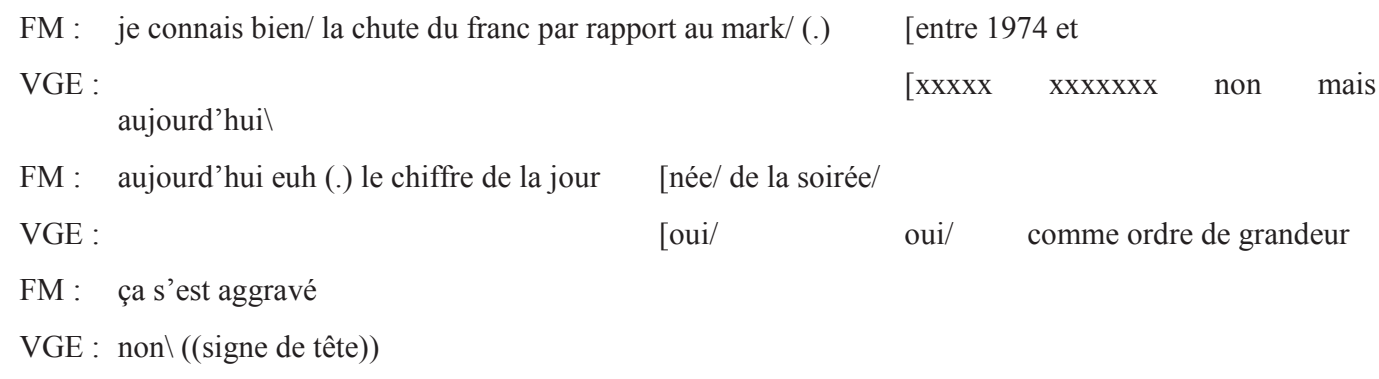

FM : d'abord je n'aime pas beaucoup/ hein/ je vais vous dire les chiffres $\backslash$ (.) je n'aime pas beaucoup cette méthodel je ne suis PAS votre élève/ (.) vous n'êtes pas le président de la République ici/ (.) vous êtes simplement mon contradicteur/

VGE : oui

FM : et [j'entends/j'entends bien/

VGE : $[\operatorname{xxx} \operatorname{xxxxxxxxxx}$

FM : non/ pas de cette façon-là $\backslash$ je: n'accepte pas/ cette façon de

VGE : le fait de vous demander quel est le cours [du Deutsche Mark/

FM :

[non non non non/ pas de cette façon là/ (.) ce que je veux simplement vous dire/ c'est que lorsque l'on: passe/ de: (.) un:: un franc quatre vingt sept/ à deux francs trente cinq/ environ (.) euh en l'espace de sept ans/cela n'est pas une réussite/ pour le franc/ $[\ldots]$

Cet épisode ressemble à bien des égards au précédent : il constitue lui aussi une sorte de parenthèse métacommunicative, ouverte par le connecteur «d'abord» suggérant que l'accord sur certaines règles constitue un préalable à la bonne marche du débat; il sonne lui aussi comme un coup de semonce, renforcé par la répétition et en outre ici, par le geste accusateur de l'index pointé. Mais à l'inverse de ce qui se passe dans le précédent débat, c'est FM qui administre une leçon à VGE (ce qu'il ne se permet pas de faire en 1974 où Giscard arbore pourtant bien davantage encore un comportement de maître d'école : Mitterrand a pris depuis de l'assurance et de la hauteur, assumant pleinement la posture d'un présidentiable); et c'est cette fois VGE qui se trouve mis en position basse, et réduit à se justifier en bredouillant. Par ailleurs, la négociation ne porte pas tout à fait sur le même type d'objet : c'est encore une affaire d'ethos, mais surtout de rapport de places entre les deux partenaires de l'échange.

C'est exactement le même type de négociation qu'illustre l'exemple suivant, mais avec une nouvelle et double inversion des rôles : d'une part, Mitterrand est depuis 1981 président de la République et de façon assez cocasse, il va tenter d'infliger à son nouvel adversaire, Jacques Chirac, qui se trouve être son premier Ministre, cette attitude de condescendance qu'il reprochait sept ans plus tôt au président d'alors, Giscard d'Estaing ; corrélativement, il va se trouver, comme en 1974 mais à l'inverse de 1981, en position d'attaqué - mais aidé par la maladresse de son attaquant, il va se tirer bien plus habilement que VGE de ce mauvais pas, parvenant même à retourner la situation en sa faveur.

\subsection{8 : débat entre Jacques Chirac et François Mitterrand}

- $F M$ : «Mais vous avez tout à fait raison monsieur le Premier ministre »

De par son statut, Mitterrand se trouve occuper la position haute par rapport à son adversaire, ce qu'il va tenter de marquer par l'usage bien particulier qu'il fait du terme d'adresse. Les règles en vigueur (mais elles sont sur ce point implicites et souples) veulent en effet que les co-débatteurs s'adressent l'un à l'autre à l'aide de la formule «monsieur + patronyme», cet usage symétrique et égalitaire les catégorisant comme de simples candidats. C'est en effet le seul usage attesté dans les autres débats où l'on ne rencontre aucun titre, même dans la bouche de Mitterrand qui s'adresse pourtant à un ministre en 
1974 et à un président de la République en $1981^{11}$. C'est aussi l'usage auquel recourt Chirac dans ce débat, mais Mitterrand introduit une dissymétrie en donnant très systématiquement à son interlocuteur du « monsieur le Premier Ministre », emploi donc très " marqué », dont il éprouve d'ailleurs le besoin de se justifier au bout d'une demi-heure de débat - il en prend curieusement l'initiative, après un développement où il n'est aucunement question de ce problème particulier mais où Chirac a mis en cause la conception mitterrandienne de l'impartialité de l'état, ce qui n'est évidemment pas du goût de ce dernier qui réplique :

FM : [...] je vous ai observé pendant deux ans/ vous me donnez là un bien mauvais exemple $\backslash$ mais je ne vais pas m’engager davantage $\backslash($.$) moi je \backslash$ (.) je vous appelle/ et je n- ne fais aucune observation particulière sur votre façon de vous exprimer/ vous en avez le droit $\backslash(.) \mathrm{moi} /$ je continue de vous appeler monsieur le premier ministre/ puisque c'est comme cela que je vous ai appelé pendant (.) deux ans/ (.) et que vous l'êtrel ${ }^{12}[\ldots]$

Justifications bien évidemment fallacieuses (car il va de soi que Mitterrand est capable de faire le départ entre l'identité « objective » et l'identité « contextuellement pertinente »), qui dissimulent la vraie raison de ce choix, à savoir le souci de souligner, sous des dehors respectueux, la relation de subordination dans laquelle se trouve son adversaire par rapport à lui (JC est son premier ministre).

Chirac alors de protester : il rappelle à Mitterrand le principe même de ce débat ainsi que les identités au nom desquelles ils s'y trouvent tous deux engagés, ou plutôt celles qui sont dans ce contexte non pertinentes, «ce soir » faisant comme un écho au « ici» de la tirade mitterrandienne de 1981 (« vous n'êtes pas le président de la République ici »); puis il conclut ce rappel par un énoncé qui est en fait ambigu comme on va le voir, mais qui n'en est pas moins accompagné d'un petit sourire satisfait satisfaction prématurée étant donné ce qui advient ensuite :

JC : permettez-moi juste de vous dire que ce soir/ (.) je ne suis pas/ le Premier ministre $($.$) et vous n'êtes$ pas/ le président de la République $\backslash$ (.) nous sommes $\backslash$ (.) deux candidats/ (.) à égalité/ (.) et qui se soumettent au jugement des Français (.) le seul qui compte $\backslash$ (.) vous me permettrez donc de vous appeler monsieur Mitterrand $\backslash$

FM : mais vous avez tout à fait raison/ monsieur le Premier ministrel

Après cette répartie Mitterrand relève le buste et esquisse un petit sourire malicieux, Michèle Cotta ne parvient pas à réprimer un sourire plus marqué en jetant un regard à la ronde, pendant que s'installe un silence de plus de deux secondes avant qu'intervienne l'autre animateur, d'une façon qui laisse soupçonner quelques réactions intempestives ${ }^{13}$ mais sans faire la moindre allusion explicite à l'incident :

EV (Elie Vannie) : messieurs/ (.) messieurs/ si vous le voulez bien/ avant de donner la parole à Michèle Cotta $[\ldots]$

$\mathrm{Au}$ sujet de cette fameuse réplique mitterrandienne, Trognon \& Larrue (1994: 76) parlent de " dénégation » et d'énonciation aux allures de "paradoxe». Sans doute. Mais un examen plus attentif fait apparaître que la réplique se prête en fait à deux interprétations, selon la «portée » que l'on attribue à «vous avez tout à fait raison »: c'est une expression anaphorique, mais quel est exactement son antécédent? sur quoi « porte » véritablement l'accord?

L'interprétation la plus naturelle consiste à considérer que cette évaluation porte sur l'ensemble de la précédente réplique de Chirac, et surtout sur son noyau « nous sommes deux candidats à égalité ». Selon cette interprétation, «vous avez tout à fait raison » signifie : vous avez raison de dire que nous sommes deux candidats à égalité, ce qui implique que nous utilisions réciproquement la formule d'adresse «monsieur + patronyme ». Dans cette interprétation il y a bien contradiction entre le contenu de l'énoncé et le terme d'adresse qui l'accompagne : c'est une boutade, qui suscite le sourire ou le rire.

Mais en même temps, Mitterrand protège ses arrières : il peut s'innocenter de ce scandale discursif que constitue la contradiction en se réfugiant derrière une autre interprétation. En effet, «vous avez tout à fait raison » peut aussi être entendu comme ne portant que sur la fin du tour précédent, c'est-à-dire «vous me permettrez donc de vous appeler monsieur Mitterrand», qui signifie à la lettre : «je 
continuerai si vous le permettez de vous appeler monsieur Mitterrand» (sans rien dire de la réciproque) : vous avez raison de faire comme cela vous chante, en ce qui me concerne je continuerai à vous appeler comme il me sied - et de fait, Mitterrand maintiendra tout au long du débat son recours au titre ${ }^{14}$, quant à Chirac il restera fidèle à «monsieur Mitterrand», sauf vers la fin où il laissera échapper un «monsieur le président» peut-être chargé d'une connotation ironique («ah mais moi je n’ai pas changé moi j'applique cette politique depuis deux ans monsieur le Président »).

De ces deux interprétations, la première est assurément plus conforme au fonctionnement général de l'anaphore, et s'impose davantage (comme en témoignent la réaction que suscite à chaud cette boutade et les nombreux commentaires postérieurs). La deuxième interprétation est plus douteuse, pour des raisons aussi bien syntaxiques que sémantiques : le segment «vous me permettrez donc... » apparaît comme la conclusion de l'ensemble du raisonnement que constitue la réplique de Chirac et peut donc difficilement être isolé pour servir de base à l'enchaînement (d'autant plus qu'il s'agit d'un segment à valeur directive et non assertive) ; en outre, la conclusion logique à laquelle aboutit ce raisonnement, c'est non seulement le sens explicite de "vous me permettrez de vous appeler Monsieur Mitterrand » mais aussi ce que l'on peut en inférer, à savoir « et de vous prier de bien vouloir m'appeler Monsieur Chirac » (il s'agit plus d'une requête indirecte qu'une simple demande de permission). Mais le malheur (pour Chirac), c'est que l'on peut toujours faire la sourde oreille aux inférences ${ }^{15} \ldots$ La deuxième interprétation n'est donc pas complètement exclue, surtout si l'on prend en compte le cotexte antérieur : elle va en effet dans le sens de la remarque faite par Mitterrand dans le tour précédent où il devance pour ainsi dire le reproche de Chirac («je ne fais aucune observation particulière sur votre façon de vous exprimer, moi je continue de vous appeler monsieur le premier ministre puisque...»).

Ainsi Mitterrand fait-il d'une pierre deux coups : il construit de lui-même l'image d'un débatteur tolérant et « non formaliste », pour reprendre le mot qu'il utilise lui-même un peu plus tôt dans le débat (alors que les raisons de cette préférence appellative sont évidemment moins innocentes), tout en divertissant l'auditoire. Et surtout, il joue un bon tour à son adversaire, victime de la maladresse de sa formulation.

\subsection{5 : débat entre Jacques Chirac et Lionel Jospin}

- $L J$ : «Il vaut mieux cinq ans avec Jospin que sept ans avec Jacques Chirac»

Considéré comme le plus technique et courtois, donc ennuyeux, ce débat comporte toutefois un moment d'humour d'autant plus inattendu qu'on le doit à Jospin, qui ne passe pas spécialement pour un joyeux drille. Il survient au cours de la séquence concernant la question de la réduction à cinq ans du mandat présidentiel, à laquelle Jospin se déclare favorable alors que Chirac se montre beaucoup plus réticent ( « je considère que le quinquennat n'est pas d'actualité »), s'en prenant à la manie française de changer les textes «toutes les deux ou trois décades ». Le terme de « décade » étant manifestement impropre, Jospin se permet quelques minutes plus tard de le rectifier « mine de rien » (car rien dans son expression ne vient signaler ce qui constitue pourtant une petite insolence), puis il poursuit de la façon suivante son argumentation en faveur du quinquennat :

LJ : $\quad[\ldots]$ c'est une question qui est pendante/ pour lequel on a un texte qui existe et qui a été voté dans les mêmes termes par DEUX assemblées $\backslash$ qui a été proposé/ (.) il y a maintenant deux décennies $\backslash$ et non pas deux décades/ mais deux décennies/ (.) par eu:h quelqu'un qui fut votre mentor en tout cas un homme que vous avez euh beaucoup connu et respecté/ (.) Georges Pompidou $\backslash$ (.) et donc (.) moi je suis le premier à dire (.) si je suis élu par les Français/ je proposerai/ (.) cette réforme/ (.) car je pense que dans une fonction d'une telle responsabilité que celle de la présidence de la République (.) c'est trop long que sept ans à mon avis surtout si ce mandat peut être renouvelable $\backslash$ (.) en somme je voudrais dire en en en badinant/ bien sûr $\backslash$ (.) mais/ avec un fond de sérieux $\backslash$ (.) qu'il vaut mieux cinq ans avec Jospin (.) que sept ans/ (.) avec Jacques $\quad$ [Chirac $\backslash$ hein/ ça serait BIEN long $\backslash$ ça serait bien long $\backslash$

$\mathrm{JC}$ :

$[(($ rires $))$ 
Sans renoncer complètement à son «sérieux », Jospin s'essaie donc au registre «badin ». Bien qu'annoncée par une formule destinée à en amortir l'audace (et encore adoucie par le sourire qui l'accompagne), cette semi-boutade tire d'abord son effet de l'emploi de la troisième personne : tout se passe comme si Jospin se dédoublait pour observer de l'extérieur les deux candidats mis en balance. Mais son efficacité vient aussi de ce qu'elle opère une sorte de condensation argumentative, puisqu'elle fusionne en un seul deux énoncés comparatifs en principe indépendants (mais qui ne le sont pas tant que ça étant donné que Jospin se dit favorable au quinquennat alors que Chirac prône le maintien du septennat) : d'une part «il vaut mieux un quinquennat qu'un septennat », idée que Jospin s'emploie à défendre tout au long de ce passage, et «il vaut mieux Jospin que Chirac », idée qui sous-tend l'ensemble des propos du candidat tout au long du débat mais que le bon goût interdit de formuler directement.

Beau joueur, Chirac accueille ce trait d'esprit en éclatant d'un rire franc. Après cette incartade, Jospin réintègre pour de bon le registre sérieux. Tranchant sur la grisaille du débat, cette " petite phrase »qui serait d'après Delporte (2012: 297) «la seule de la soirée »—est d'autant plus « marquante » qu'elle tranche aussi sur le comportement attendu de son auteur (l'ethos affiché venant localement remodeler l'ethos préalable).

Le débat de 2007 fut assurément plus mouvementé.

\subsection{7 : débat entre Ségolène Royal et Nicolas Sarkozy}

- $S R$ : «Je ne suis pas énervée, je suis en colère »

Le dernier cas envisagé se différencie des quatre autres d'abord par sa longueur : il ne s'agit pas d'une « petite phrase » décochée par l'un des débatteurs afin de mettre à mal son adversaire, mais d'un épisode conflictuel relativement étendu. Il survient aux deux tiers environ de ce débat exceptionnellement long (2h40), au sein duquel il constitue une sorte de pièce détachée par son thème (cette séquence est entièrement focalisée sur l'état émotionnel de Ségolène Royal) mais aussi par la présence en son début et en sa fin d'énoncés à fonction clairement démarcative. Pour ce qui concerne l'ouverture : alors qu'est censée s'achever la séquence sur la politique économique et sociale, Sarkozy vient de vanter le dispositif du «droit opposable», et pour contrer les sarcasmes de Royal il prend l'exemple (un « exemple qui va peut-être vous toucher», annonce-t-il — il ne croit pas si bien dire...) des enfants handicapés qui se voient refuser l'accès dans les écoles, dont il conclut (et l'intonation comme la mimique viennent souligner la valeur conclusive de cette sorte de péroraison) que c'est ce genre de mesure «qui fait la différence entre la vieille politique et la politique moderne ». Les animateurs (Patrick Poivre d'Arvor et Arlette Chabot) félicitent alors les débatteurs d'avoir «réussi la prouesse de...», mais on ne saura jamais exactement de quoi car Royal les interrompt avec un solennel « attendez j'ai quelque chose à dire » qui sonne comme un coup de théâtre.

Ce qu'elle a à dire, c'est qu'elle est «scandalisée » par l' «immoralité politique » dont fait preuve Sarkozy en tenant un discours «larmoyant» sur les handicapés alors que c'est justement lui qui a «cassé le plan Handiscole» qu'elle avait elle-même mis en place lorsqu'elle était ministre de l'enseignement scolaire. Elle l'accuse donc d'un «écart entre le discours et les actes » et conclut son réquisitoire en se déclarant «très en colère » (auto-attribution explicite d'un état émotionnel assumé et même revendiqué). Après l'avoir laissé parler pendant plus de deux minutes, Sarkozy l'interrompt pour lui demander de se calmer, ce à quoi Royal rétorque qu'elle ne se calmera pas («non je ne me calmerai pas » répété quatre fois : elle persiste et signe) car il y a des colères qui sont saines "parce qu'elles correspondent à la souffrance des gens $»$ :

$\begin{array}{lll}\mathrm{SR}: & {[\ldots] \text { je suis très en colère } \backslash(.) \text { et les parents et les familles qui vous }} & \text { [ent-\& } \\ \mathrm{NS}: & & {[\underline{\text { calmez-vous }}} \\ \mathrm{SR} \text { : } & \text { \&et les parents }\end{array}$

SR : \&et les parents [non je ne me calmerai pas

NS : $\quad[\underline{\text { calmez-vous et ne me montrez pas du doigt } \quad[\text { avec ce\& }}$ 
NS : \&cet index [pointé parce que franchement (.) je je je voudrais vous di- je voudrais

[je ne me calmerai pa:s (.) parce que: l'exploit-

NS : [ben pour être président de la République i faut être calme

SR : $\quad$ non: (.) pas quand il y a des injustices $\backslash$ (.) il y a des colères qui sont parfaitement [sai:nes (.)\&

NS : $\quad[$ bon

SR : \&parce qu'elles correspondent [à la souffrance des gens\ (.) il y a des colères que j'aurai même quand je serai présidente de la République $\&$

NS :

[madame Royal (.) est-ce que vous me permettez de vous dire un mot

SR : $\quad \&[($.$) parce que je (.) parce que je$

NS : $\quad$ [eh ben ça sera gai (.) ça sera gai

$[\ldots]$

NS : $\quad$ je je je ne (.) je ne sais pas pourquoi euh madame Royal euh d'habitude calme a perdu

[ses nerfs (.) parce que (.) parce que j'ai

SR : $\quad$ non je ne perds pas mes nerfs je suis en colère $\backslash$ (.) ce n'est pas pareil pas de mépris monsieur $\underline{\text { Sarkozy } \backslash \text { (.) pas de mépris }}$

Dans ce passage, on assiste en direct à la construction conflictuelle de deux micro-systèmes lexicaux partiellement divergents : SR opère au sein de la notion de «colère » une dissociation entre la colère « saine » et les autres formes de colère (dont il ne sera pas question), mettant en place la notion de « saine colère » qu'elle définit (les saines colères sont celles qui procèdent d'un sentiment de révolte devant le spectacle de la souffrance), qui est pour elle chargée d'une valeur axiologique positive, et qui $s$ 'oppose en tout point à la notion d'énervement : « non je ne perds pas mes nerfs je suis en colère (.) ce n'est pas pareil pas de mépris monsieur Sarkozy » - le mépris consistant en l'occurrence dans le fait de ravaler une émotion noble et réfléchie au rang d'un vulgaire coup de sang incontrôlé. C'est en effet ce à quoi s'emploie de son côté NS tout au long de la séquence : il assimile colère et énervement, utilisant successivement comme de simples variantes les expressions "perdre ses nerfs », "s'énerver», « sortir de ses gonds », « se mettre en colère » et «perdre son sang-froid» : toutes ces expressions sont pour lui synonymes et également chargées dans ce contexte d'une connotation négative.

Cette négociation sur le sens des mots est corrélative d'une négociation sur le référent auquel ils s'appliquent, à savoir l'état émotionnel de SR : faut-il le catégoriser comme un état de colère ainsi que le revendique SR elle-même, ou comme un état d'énervement ainsi que le prétend Sarkozy, pour qui les deux états reviennent au même, et sont de toute façon contraires à l'ethos d'un bon président de la République ? Car tel est finalement l'enjeu de ce débat : la «présidentiabilité » de SR ; point crucial qui lui aussi donne lieu à un désaccord entre les deux protagonistes, NS répétant que «pour être président de la République il faut être calme [or vous ne l'êtes pas, donc...] » alors que SR proclame de son côté qu'un bon président doit être capable de temps en temps de piquer des colères, saines bien sûr (« il y a des colères que j'aurai même quand je serai présidente de la République »). Cet enjeu est lourdement souligné par Sarkozy en ces termes :

NS au moins ça a eu une utilité madame $($.$) c'est que vous vous mettez bien facilement en colère ($. vous sortez de vos gonds avec beaucoup de facilité madame (.) président de la république/ (.) c'est quelqu'un qui a des responsabilités lourdes (.) très lourdes $\backslash$ 
- autrement dit : cet épisode apparemment vain a au moins permis de vous démasquer comme colérique (vous avez été prise en flagrant délit de «non-calmitude»), donc de vous disqualifier dans votre prétention à la fonction suprême.

Ce que SR ne peut évidemment pas laisser passer : l'importance de l'enjeu explique l'exceptionnelle durée de la séquence (qui s'étale sur plus de huit minutes alors que la modératrice a accordé à Royal " un mot puis on enchaîne »). La négociation piétine et s'éternise, offrant diverses variations sur le thème « Ne vous énervez pas - Je ne suis pas énervée, je suis en colère ». Les animateurs tentent par trois fois, mais sans grand succès, de fermer cette parenthèse et de lancer le thème de l'Europe. Au moment même où il semble qu'ils y soient enfin parvenus, Sarkozy revient machiavéliquement à la charge :

NS non: (.) pis je (.) et je vais même vous dire quelque chose [...] je vous en veux pas parce que ça peut arriver à tout le monde de s'énerver)

et c'est reparti pour un dernier petit tour, car Royal ne peut une fois encore que protester «je ne m'énerve pas je me révolte car j'ai gardé ma capacité de révolte intacte », mais ce définitif «mot de la fin » se trouve noyé dans un brouhaha général. En un tournemain, elle change alors à la fois de ton, de thème et de posture pour se lancer, après une sorte de petit rire qui marque ce radical changement de footing $^{16}$, dans un discours sur l'Europe car n'est-ce pas, l'Europe «c'est très important » - fin de l'épisode émotionnel, mais non des commentaires sur cet épisode, qui iront bon train dès le lendemain du débat.

\section{Bilan}

\subsection{Des épisodes « marquants ॥}

Sans prétendre élucider les raisons du succès de ces divers épisodes, on dira que leur caractère «marquant» vient de ce qu'ils se «démarquent» de la routine du débat, tout en contribuant efficacement à la réalisation de l'objectif principal de tout débatteur: marquer des points sur l'adversaire.

D'une part, toutes les séquences analysées apparaissent comme des sortes de pièces détachées par rapport à leur environnement immédiat : elles sont encadrées par des unités à fonction démarcative, elles instaurent une rupture par leur contenu (le débat s'interrompt provisoirement pour laisser la place à quelque mise au point à caractère métacommunicatif), mais aussi par leur ton et leur style (élaboration rhétorique particulière à fonction ludique ou pathétique, irruption des affects ou dramatisation de l'échange.... $)^{17}$.

Mais si rupture il y a, ces interventions ne s'en inscrivent pas moins dans le droit fil du débat par leur contenu thématique (dans un tel contexte, toute digression patente serait sévèrement sanctionnée) comme par leur visée pragmatique : mettre à mal l'adversaire, le plonger dans l'embarras, lui clouer le bec, lui donner une leçon, le remettre à sa place... — bref, remporter sur lui une victoire locale mais par des moyens plus astucieux que brutaux (il s'agit de porter l'estocade sans recourir à un coup bas), par quelque « trouvaille » qui force l'admiration du public par son « à propos » tout en déstabilisant le rival.

Créant un effet de surprise sans pour autant apparaître comme "gratuites", ces interventions contribuent surtout à la construction d'une image favorable, et parfois inédite (Giscard compassionnel, Jospin badin...) de leur auteur, envisagé toutefois — c'est là leur limite - moins en tant qu'homme politique crédible que comme un débatteur habile. 


\subsection{Analyse du discours et interprétation}

Revenons à présent sur notre question initiale: que fait-on exactement quand on « analyse du discours »?

On scrute attentivement la matière qui compose les échantillons à décrire, mais pour en extraire quoi ? Face à la séquence de la « saine colère » par exemple, il va de soi qu'on ne peut pas se contenter d'une paraphrase du genre « il lui dit de ne pas s'énerver, elle répond qu'elle n'est pas énervée mais qu'elle est en colère ", mais qu'il nous faut interpréter ce qui se passe. Mais en quoi consiste au juste cette activité, et en quoi diffère-t-elle selon que le discours auquel on a affaire a ou non un caractère interactif ?

\subsubsection{Les principes d'availability et d'accountability}

D'après Schegloff (1997: 183-184), le talk-in-interaction offre à l'interprétation une «prise » (leverage), un point d'appui plus stable que ne le fait le discours monologal, étant donné que le sens des énoncés qui s'échangent est «embodied and displayed» dans et par le comportement mutuel des interactants, lesquels se rendent mutuellement accessible le sens qu'il convient d'assigner aux énoncés et activités qu'ils déploient tout au long du déroulement de l'interaction. Dans la perspective de l'analyse conversationnelle, décrire un échantillon de discours-en-interaction revient donc à recueillir ces faits pertinents rendus disponibles (available) et reconnaissables (accountable) par et pour les participants à l'interaction — mais aussi, du même coup, pour les observateurs extérieurs cherchant à la décrire :

[Les faits pertinents] sont rendus publiquement disponibles dans des conduites reconnaissables comme telles [...] (Gülich et Mondada, $2001: 208$; italique ajouté)

La conversation s'appuie sur un façonnage organisé de traits dont le sens est rendu public et accessible en surface pour les participants à des cours d'action. Ce dispositif de publicité [...] (Relieu et Brock, 1995 : 79 ; italique ajouté)

Mais ce qui est « rendu public », ce sont des signifiants, qui ne sont pas transparents : il ne suffit pas de regarder, même très attentivement, les données que l'on a collectées pour voir miraculeusement surgir leur sens. Revenons par exemple sur le « vous avez tout à fait raison monsieur le Premier ministre » de Mitterrand : sans doute le petit sourire qui souligne malicieusement la fin cet énoncé signale-t-il quelque chose - mais quoi ? Il ne nous précise en rien que l'énoncé en question peut être interprété de deux façons, dont l'une présente une contradiction interne, et qu'il s'agit donc d'une «boutade »; ce qui ne peut apparaître qu'au terme de tout un travail d'analyse, censé reconstituer celui qu'ont effectué les participants eux-mêmes, sans qu'il soit au demeurant possible de savoir comment cette déclaration a été véritablement reçue par son destinataire principal, Jacques Chirac. Quant à Michèle Cotta, le sourire qu'elle ne parvient pas à réprimer nous suggère qu'elle a été sensible à ce trait d'esprit, mais l'observation de cette réaction ne saurait se substituer à l'analyse de ce qui la suscite. Plus généralement, la description d'un segment quelconque de discours-en-interaction ne saurait se réduire à l'enregistrement de la façon dont ce segment est traité par le next speaker (qui ne traite qu'une infime partie du matériel qui lui est soumis - en particulier dans ces débats qui comportent de longues tirades monologuées - , et dont la réaction doit elle-même être soumise à interprétation) ${ }^{18}$.

En réalité, le principe d'accountability ne fait que reformuler le principe sémiotique : chaque participant délivre des signifiants (qu'on les appelle cues, marqueurs, indicateurs ou « traits») à l'intention d'autrui, qui n'a plus qu'à les interpréter... Ce qui engage en fait une activité complexe mobilisant toutes sortes de savoirs (sur la langue, le fonctionnement de la communication, les règles propres au genre interactionnel, le contexte étroit et large, etc.), l'analyste ayant pour tâche de reconstituer après coup le travail effectué en temps réel par les participants, ce qui n'est possible qu'à la condition qu'il dispose des mêmes savoirs et aptitudes (que l'on parle à ce sujet de «ressources» ou de « compétences »). 


\subsubsection{Adopter le point de vue des participants}

Etant admis que l'analyste doit ajuster son point de vue à celui des participants à l'interaction, le problème se pose de savoir ce qu'il faut entendre par "participants », étant donné la complexité du format participatif (format de production et surtout de réception) qui caractérise les interactions médiatiques en général, et ces débats en particulier. Or si l'on peut admettre que tous les participants disposent à peu près de la même compétence linguistique et plus largement communicative, il n'en est pas de même en ce qui concerne leur compétence « encyclopédique », pourtant fortement mobilisée au cours du processus interprétatif. L'analyste se trouve donc confronté à la question suivante : est-il légitime de prendre en compte certains éléments relevant du background de tels ou tels membres, mais d'eux seuls? Par exemple, en ce qui concerne les débatteurs eux-mêmes: dans le débat de 1974, Giscard lance sans ciller à Mitterrand, en plein milieu d'un développement sur «la France qui travaille »:

Clermont-Ferrand/ est une ville/ qui a une des plus grandes/ usines/ de France $\backslash$ (.) et qui a une municipalité/ socialistel (.) et c'est une ville/ qui vous connaît bien/ (.) et qui me connaît bien $\backslash($.

Ce que Delporte commente ainsi (2012 : 133, n.6) :

Giscard d'Estaing use d'une allusion codée à la double vie de Mitterrand, destinée à le déstabiliser, la famille d'Anne Pingeaud étant installée à Clermond-Ferrand.

On ne voit pas au nom de quoi il faudrait dénier à cette donnée toute pertinence analytique, bien qu'elle ne soit accessible qu'à un tout petit nombre de participants.

En ce qui concerne les animateurs : chargée avec Patrick Poivre d'Arvor de modérer le débat de 2007, Arlette Chabot déclara plus tard qu'elle avait vécu cette expérience comme extrêmement frustrante par rapport à la conception qu'elle se faisait de son rôle de journaliste, et même comme un « cauchemar ». Une analyse fine du déroulement de ce débat permet en effet d'expliquer cette impression et confirme que l'animatrice est plus ou moins traitée comme une " potiche » (pour reprendre son propre terme) par les débatteurs mais aussi par le co-animateur ${ }^{19}$ - le plus troublant étant que cet état de fait, bien qu'incontestablement inscrit dans le texte même de l'interaction, n'est pas immédiatement perceptible lorsque l'on visionne le corpus.

Reste le problème de la masse des télespectateurs, citoyens ordinaires ou politologues experts, qui ne sont pas en mesure d'intervenir dans le débat mais ne se priveront pas de le commenter après coup. Il semble difficile de ne pas les considérer comme des "membres» alors qu'ils sont les principaux destinataires de ce qui se passe sur le plateau (c'est aussi et même surtout pour eux que les propos tenus par les débatteurs doivent être accountable), ainsi que le rappellent parfois les débatteurs eux-mêmes, par exemple Giscard en 1974 :

dans ce débat/ il y a quelque chose que nous n'essaierons pas de faire/ qui est de nous convaincre/ mutuellement $\backslash[. .$.$] ce que nous devons faire/ c'est d'éclairer le$ choix/ des Françaises/ et des Français $\backslash$ (.)

auquel Mitterrand fait peu après écho :

je crois que nous sommes ici aussi/ pour/ (.) informer $\backslash$ pour faire comprendre $\backslash$ (.) pour dialoguer $\backslash$ (.) avec/ la France $\backslash$ (.) et donc avec les Français $\backslash$

Mais le problème est évidemment que l'on ne dispose d'aucune trace du travail interprétatif effectué par cet auditoire très hétérogène, ni de ses résultats, si ce n'est après coup. Sans entrer dans la problématique des recherches sur la réception ${ }^{20}$, on admettra qu'il n'est pas interdit à l'analyste de discours de jeter un coup d'œil sur les nombreux commentaires, circulant dans les médias ou sur le Net, des épisodes qui l'intéressent, afin de voir comment ils ont été reçus et perçus — éventuellement en contradiction avec le «diagnostic » qu'il/elle a été amené(e) à effectuer. S'agissant par exemple de l'épisode de la "saine colère », on peut au terme de la description détaillée de la séquence se demander si finalement, Ségolène Royal se montre « en colère » (comme elle l'affirme elle-même) ou « énervée » 
(comme le prétend Sarkozy). Une analyse minutieuse de son comportement aussi bien verbal que non verbal invite à penser que non, elle n'est pas «sortie de ses gonds». Pourtant, nombreux sont les commentateurs de cet épisode, qu'ils soient profanes ou experts ès discours politiques, qui à l'encontre de ce diagnostic ont parlé et parlent encore du «moment où Ségolène Royal s'est énervée », épousant sans état d'âme l'interprétation sarkozienne, quel que soit au demeurant leur penchant politique. Or il est permis de penser que l'analyste n'a pas à traiter par le mépris ces interprétations « erronées » (à partir du moment où elles ne sont pas isolées) et que le texte interactionnel, en relation avec certaines données contextuelles, doit bien être dans une certaine mesure responsable de ces effets de sens. On peut en l'occurrence alléguer divers facteurs favorisant cette interprétation. Il y a d'abord le fait qu'à force de marteler «ne vous énervez pas », Sarkozy a fini par convaincre la masse des spectateurs-auditeurs que Royal était effectivement énervée (c'est du moins ce qui s'est inscrit dans les mémoires). Il y a aussi le fait que le distinguo, légitime sans doute, que tente d'établir Royal entre deux affects qui sont tout de même proches est sans doute trop subtil dans un tel contexte (il ne s'agit pas d'un débat philosophique de France Culture) : la langue ordinaire admet bien une sorte de synonymie entre le fait d'être « en colère » et celui d'être «énervé »; la position de Sarkozy est donc plus conforme au sens commun que celle de Royal, plus conforme aussi à la représentation prototypique de la colère (impliquant une certaine violence et entraînant un certain dérèglement du comportement) — sans parler du stéréotype de la « femme hystérique » qui joue évidemment un certain rôle dans cette affaire ${ }^{21}$. Il y a enfin l'excessive durée de la séquence : Royal s'acharne, répétant ad nauseam «je ne m'énerve pas je suis en colère »et l'on a vu pourquoi elle refusait de lâcher le morceau : c'est que c'est son ethos présidentiel qui est en jeu. Mais en s'obstinant de la sorte elle risque de susciter l'exaspération du public qui a vraiment envie que l'on passe à autre chose, et que s'achève cette parenthèse qui donne l'impression de se faire au détriment des problèmes de fond, comme si ce numéro quelque peu factice et théâtral dans lequel Royal joue les pasionarias offusquées n'était qu'une sorte de cache-misère. Il semble donc qu'en se laissant entraîner dans cette trop longue digression psychologico-éthique Ségolène Royal soit bel et bien tombée dans le piège tendu par son adversaire.

Pour un dernier exemple de la façon dont les représentations préalables (il s'agit en l'occurrence de savoirs intertextuels) déterminent l'interprétation des énoncés, revenons sur la question du « monopole du cœur ». De par son caractère de « formule » (au sens de Krieg-Planque 2009), l'expression se prête à toutes sortes de réemplois, comme celui auquel se livre, en une sorte de petite revanche ironique, Mitterrand contre Giscard en 1988 (à propos de l'augmentation de la TVA sur les aliments pour chiens et chats) :

FM : [...] vous avez parlé des chats et des chiens/ moi aussi je les aime beaucoup/ d'ailleurs je crois que nous avons (.) des chiens de la même espèce/ et (.) dieu sait si on s'y attache/ (.) alors euh $\backslash$ (.) j’peux pas vous répéter ce que j'avais entendu naguère/ (.) vous n'avez pas le monopole du cœur pour les chiens et les chats/ (.) je les aime moi aussi/ [...]

Mais on peut aussi voir se profiler la formule, bien qu'elle n'y figure pas au complet, dans ce passage du débat de 2007 où elle serait tout à fait à sa place :

NS : [...] donc j'ai le droit de parler du handicap/ (.) ce n'est pas le monopole (.) qui est le vôtre/ (.) j'ai le droit d'être sincère dans mon engagement/ (.) et d'être bouleversé (.) par la situation d'enfants qui aimeraient être scolarisés/ [...]

D'un point de vue grammatical, la phrase est quelque peu bancale : peut-être peut-on voir dans ce fait, ainsi que dans la pause qui suit l'énoncé du mot «monopole», la trace d'une sorte de problème énonciatif; lequel ne se précise qu'à la lumière d'une information externe : peu auparavant en effet, lors d'un discours prononcé au Mont-Saint-Michel, Sarkozy avait cité la phrase de VGE mais en l'attribuant malencontreusement... à Mitterrand. Ainsi outillé, l'analyste peut faire l'hypothèse que cette construction bizarre s'expliquerait par une sorte de refoulement de la formule du « monopole du cœur », associée pour Sarkozy au souvenir de cette «boulette», dont la presse n'avait pas manqué de se gausser. 
Toute interprétation doit s'ancrer dans la matérialité textuelle, et reposer sur la présence de marqueurs ou d'indices - mais une fois ce beau principe rappelé, il ne permet pas toujours de répondre, face à des cas concrets, à ces trois questions indissociables : ceci est-il un indice ? de quoi ? et pour qui ?

\subsubsection{L’analyste comme " archi-interprétant »}

Quel que soit le type de discours auquel on a affaire, il revient à l'analyste de tenter de reconstituer la façon dont le texte et le contexte sollicitent certaines interprétations. Dans le cas d'un discours monologal (par exemple, une conférence magistrale ou un article de journal), la tâche consiste à voir sur quoi repose et en quoi consiste l'intelligibilité de ce discours pour son auditoire ou son lectorat. Dans le cas d'un discours dialogué, il s'agit de voir comment les différents participants se rendent mutuellement intelligibles les énoncés qu'ils échangent, en « négociant » à l'occasion l'interprétation qu'il convient de leur attribuer, interprétation qui sert de base à l'enchaînement. Le cas de nos débats est particulièrement complexe car ils relèvent à la fois de ces deux cas de figure, étant donné que les échanges qui se déroulent sur le plateau (communication à double sens) se destinent en réalité au collectif des téléspectateurs (communication à sens unique).

Dans tous les cas de figure, cette reconstitution nécessite que l'analyste dispose des mêmes «ressources » que celles qui ont été mobilisées par les participants ${ }^{22}$, mais aussi qu'il soit en mesure d'expliciter le processus qui lui permet d'assigner la/les valeur(s) que reçoit tel ou tel segment pour tel ou tel participant. Ce travail d'explicitation ne peut se faire sans le recours à un outillage descriptif diversifié, dont on a vu qu'il pouvait être emprunté à la syntaxe ou à la sémantique aussi bien qu'à la rhétorique, la pragmatique, ou l'analyse conversationnelle.

On notera pour conclure que quelles que soient les innovations introduites, par l'attention enfin portée au fonctionnement des interactions orales, dans le champ l'analyse du discours, on y rencontre toujours, même s'ils ne sont plus formulés exactement dans les mêmes termes, les mêmes questionnements théoriques fondamentaux - par exemple et entre autres :

Étant donné que le sujet discoureur ne «publie» jamais que des signifiants, et que le passage au signifié implique toujours un certain «saut interprétatif», qu'est-ce qui peut garantir la validité de l'exercice ? et quelle part raisonnable de risque peut-on y admettre ?

Quelle place convient-il d'accorder dans la description aux informations « externes »? (le vieux débat entre les partisans d'une approche «immanentiste» et ceux qui dénoncent l'«illusion endogène » connaissant aujourd'hui de nouveaux rebondissements dans le champ de l'analyse des interactions).

Étant donné que tout fragment de discours est produit et interprété sur la base d'un système de normes et de conventions préexistantes (linguistiques et non linguistiques), dans quelle mesure peut-on dire qu'il « recrée », voir « crée », ses propres règles de fonctionnement ? ? $^{23}$

Enfin, quelle est l'importance relative des similitudes et des spécificités propres à chaque type de discours et corrélativement, dans quelle mesure les méthodologies adoptées doivent-elles être communes ou différenciées ? ${ }^{24}$

Autant de questions qui n'ont toujours pas trouvé de réponse définitive, et c'est tant mieux.

\section{Principales conventions de transcription}
/: intonation montante
$\backslash$ : intonation descendante
[ : chevauchement de parole
(.) : pause 
Majuscules : segment accentué

Xxx : segment inaudible

\& : poursuite du même tour de parole

\section{Références}

Agha, A. (1997). Tropic aggression in the Clinton-Dole presidential debate. Pragmatics 7(4), 461-497.

Bert, M. et al. (2010). Grands corpus et linguistique outillée pour l'étude du français en interaction (plateforme CLAPI et corpus CIEL). Pratiques 147-148, 17-34.

Bertrand, D., Dezé, A. et Missika, J.-L. (2007). Parler pour gagner. Sémiotique des discours de la campagne présidentielle de 2007. Paris : Presses de la Fondation Nationale des Sciences Politiques.

Bouchard, R. (2005). Le “cours”, un événement oralographique structuré. Le français dans le monde, 64-74.

Burger, M. et Martel, G. (éds) (2005). Argumentation et communication dans les médias. Québec : Nota bene.

Burger, M., Jacquin, J. et Micheli, R. (éds) (2011). La parole politique en confrontation dans les médias, Bruxelles : De Boeck.

Constantin de Chanay, H. et Kerbrat-Orecchioni, C. (2010). Les interruptions dans les débats médiatiques : une stratégie interactionnelle. Pratiques 147/148, 83-101.

Constantin de Chanay, H., Giaufret, A. \& Kerbrat-Orecchioni, C. (2011). La gestion interactive des émotions dans la communication politique à la télévision: quand les intervenants perdent leur calme. In Burger et al. (éds) $2011,25-50$

Delporte, C. (2012). Les grands débats politiques. Paris : Flammarion/INA.

Doury, M. (2009). "Sans faire allégeance" : l'euphémisme dans l'expression des accords et désaccords dans un débat politique. Synergies Italie (ํㅗㄹ spécial « Euphémismes et stratégies d'atténuation du dire »), 111-122.

Doury, M. et Kerbrat-Orecchioni, C. (2011). La place de l'accord dans l'argumentation polémique : le cas du débat Sarkozy/Royal ». A contrario 16, 63-87 ; édition numérique : http://www.cairn.info/revue-a-contrario-20112.htm

Fish, S. (2007). Quand lire c'est faire. L'autorité des communautés interprétatives. Paris, Les prairies ordinaires.

Goffman, E. (1981). Forms of Talk. Oxford : Blackwell.

Gülich, E. et Mondada, L. (2001). Konversationsanalyse. In G. Holtus, M. Metzeltin et C. Schmitt (eds), Lexicon der Romanistischen Linguistik, vol. I-2. Tübingen : Niemeyer, 196-252.

Have, P. ten (1998). Doing Conversation Analysis. London : Sage.

Hutchby, I. (2005). Conversation Analysis and the Study of Broadcast Talk. In K.L. Fitch et R.E. Sanders (eds), Handbook of Language and Social Interaction. Mahwah (N.J.)/London : Lawrence Erlbaum, 437-460.

Kerbrat-Orecchioni, C. (2005). Le discours en interaction. Paris : A. Colin.

Kerbrat-Orecchioni, C. (2012, à paraître). Animer un débat électoral : l'exemple du duel Sarkozy-Royal (2 mai 2007). Bulletin suisse de linguistique appliquée 94.

Kerbrat-Orecchioni, C. (éd.) (2010). S'adresser à autrui. Les formes nominales d'adresse en français. Chambéry : Université de Savoie.

Krieg-Planque, A. (2009). La notion de "formule" en analyse du discours. Cadre théorique et méthodologique. Besançon : Presses Universitaires de Franche-Comté.

Levinson, S. (1983). Pragmatics. Cambridge : CUP.

Luginbühl, M. (2007). Conversational violence in political TV debates: Forms and functions. Journal of Pragmatics 39(8), 1371-1387. 
Mondada, L. (2001). Pour une linguistique interactionnelle. Marges linguistiques 1, http://www.margeslinguistiques.com

Relieu, M. et Brock, F. (1995). L'infrastructure conversationnelle de la parole publique. Politix 31, 77-112.

Sandré, M. (2011). Rires et sourires en politique. Mots 96, 13-28.

Schegloff, E.A. (1997). Whose text ? Whose context ?. Discourse \& Society, 8(2), 165-187.

Sullet-Nylander, F. et Roitman, M. (2010a). Voix de campagne présidentielle: quelques observations sur la question et la réfutation dans le débat télévisé Royal-Sarkozy. In M. Abecassis et G. Ledegen (éds), Les voix des Français à travers l'histoire, l'école et la presse. Oxford : Peter Lang, 303-317.

Sullet-Nylander, F. et Roitman, M. (2010b). De la confrontation politico-journalistique dans les grands duels politiques télévisés : questions et préconstruits. Actes du IIIème colloque Le Français parlé dans les médias : Les médias et le politique (Lausanne, 1-4 septembre 2009), http://www.unil.ch/clsl/page81503.html

Trognon, A. et Larrue, J. (1994). Les débats politiques télévisés. In A. Trognon \& J. Larrue (éds) Pragmatique du discours politique, Paris : A. Colin, 55-126.

${ }^{1}$ On sait que Chirac refusa de rencontrer Jean-Marie Le Pen, finaliste comme lui en 2002.

${ }^{2}$ Le terme est à prendre ici au sens faible : nos données ne sont en rien comparables aux interactions complexes où s'entremêlent toutes sortes d'activités verbales et non verbales.

${ }^{3}$ Voir par exemple Bert et al., $2010: 21$.

${ }^{4}$ Sur les divers types de négociations conversationnelles, voir Kerbrat-Orecchioni, 2005 : chap.2 ; et sur la différence entre « dialogal » et « dialogique », ibid : 16-19.

${ }^{5}$ En ce qui concerne nos débats, on trouve aisément dans la presse ou sur Internet des transcriptions « toutes faites ", qui restituent correctement le contenu des échanges mais sacrifient quantité de "détails » éminemment pertinents pour nous (un seul exemple : dans la transcription proposée par Libération du débat de 2007, figure moins du tiers des termes d'adresse effectivement prononcés par les candidats).

Les transcriptions utilisées ici ont été effectuées par Domitille Caillat (pour les quatre premiers débats) et Hugues de Chanay (pour le dernier), que je remercie de m'avoir permis d'en disposer.

${ }^{6}$ Les études consacrées aux débats politiques sont fort nombreuses - sur le seul cas des débats électoraux, mentionnons Bertrand \& al. 2007 ; Doury 2009; Constantin de Chanay \& Kerbrat-Orecchioni 2010 ; SulletNylander \& Roitman 2010a et 2010b ; Doury \& Kerbrat-Orecchioni 2011 ; Constantin de Chanay, Giaufret \& Kerbrat-Orecchioni 2011 ; certaines contributions dans Burger \& Martel (éds) 2005 et Burger \& al. (éds) 2011 ; ainsi que les numéros 89 (mars 2009) et 90 (juillet 2009) de la revue Mots. Voir aussi sur l'anglais : Agha 1997 et Luginbühl 2007.

${ }^{7}$ Voir par exemple le numéro 168, juin 2011, de la revue Communication \& Langages, «Les "petites phrases" en politique ».

${ }^{8}$ Pour un inventaire plus complet, voir la sélection effectuée par Hugues Nancy dans Les duels présidentiels. Toutes les "petites phrases", DVD accompagnant la série Les grands duels de l'entre-deux-tours des Présidentielles publiée en 2007 par l'INA.

${ }^{9}$ Évocation renforcée par le geste des mains croisées au niveau du cœur, geste auto-orienté sur «j'ai un cœur » puis allo-orienté sur « comme le vôtre ».

${ }^{10}$ Citée par Delaporte, $2012: 142$.

${ }^{11}$ D’une manière générale, le recours au titre est relativement rare en français, quelques situations d'interaction très particulières mises à part (voir sur ce problème Kerbrat-Orecchioni [éd.] 2010).

${ }^{12}$ Sic.

${ }^{13}$ Il semble que l'on perçoive en bruit de fond une sorte de brouhaha dont émergent quelques rires mais l'enregistrement ne permet pas de les identifier clairement. 
${ }^{14}$ En emploi appellatif ( 7 occurrences) mais aussi désignatif (9 occurrences), dans des séquences où FM « délocute » son interlocuteur, ce qui constitue un autre moyen de le rabaisser.

${ }^{15}$ On peut appliquer aux sous-entendus, inférences et autres implicatures ce que Wittgenstein dit du jeu de cachecache, ce jeu où « être caché est un plaisir, mais n'être point trouvé est une catastrophe ».

${ }^{16}$ Sandré (2011 : 17) voit dans ce rire de Royal « une sorte d'autocritique après qu'elle a écarté plusieurs fois le sujet en question ».

${ }^{17} \mathrm{Ce}$ qu'illustrent également d'autres petites phrases qui n'ont pas pu être retenues ici, comme le jeu paronomastique sur «l'homme du passé »/«l'homme du passif » de Mitterrand 1981, ou le théâtral « dans les yeux je la conteste [votre version des choses] » de Mitterrand 1988.

${ }^{18}$ Pour une critique de ce que l'on appelle parfois le «principe d'interprétation dialogique », ou la «next turn proof procedure » (Hutchby 2005), voir Kerbrat-Orecchioni, 2005 : 79-81.

${ }^{19}$ Pour le détail de l'analyse, voir Kerbrat-Orecchioni 2012.

${ }^{20}$ Voir sur cette question Hutchby, $2005: 439$.

${ }^{21}$ Notons que lors des primaires socialistes de 2012, Royal a préféré se dire à plusieurs reprises « indignée »...

${ }^{22}$ On pourrait convoquer ici la notion de «communauté interprétative » (Fish 2007), élaborée dans la perspective de l'écrit mais qui s'applique tout aussi bien aux interactions orales (on peut en effet rapprocher cette notion du principe de « virtual membership requirement», voir ten Have, $1998: 59)$

${ }^{23}$ « La langue appartient aux locuteurs — avant qu'au linguiste ; c'est le « je » qui se la réapproprie dans chaque acte d'énonciation, qui la réinvente pour mieux s'ajuster à la situation [...], recréant indexicalement la langue à chacun de ses usages. » (Mondada, $2001: 16$ )

${ }^{24}$ Par exemple, si l'on considère que les conversations et autres formes de talk-in-interaction sont radicalement différentes des autres types de discours, on opposera (à l'instar de Levinson, 1983: 286-294), l'analyse conversationnelle à l'analyse de discours, plutôt que de considérer la première comme partie intégrante de la seconde. 